BNL-81576-2008-CP

\title{
A Theoretical Study of the Two-Dimensional Point Focusing by Two Multilayer Laue Lenses
}

\author{
Hanfei Yan ${ }^{1,2}$, Jorg Maser ${ }^{2}$, Hyon Chol Kang ${ }^{2,4}$, \\ Albert Macrader ${ }^{3}$, and Brian Stephenson ${ }^{2}$ \\ Presented at the SPIE Optics + Photonics 2008 Conference \\ ${ }^{1}$ National Synchrotron Light Source II, Brookhaven National Laboratory, \\ Upton, NY 11973 \\ ${ }^{2}$ Center for Nanoscale Materials, Argonne National Laboratory, \\ Argonne, IL 60439 \\ ${ }^{3}$ Advanced Photon Source, Argonne National Laboratory, Argonne, IL 60439 \\ ${ }^{4}$ Advanced Photonics Research Institute, Gwangju Institute of Science and \\ Technology, Gwangju 500-712, Republic of Korea
}

August 10-15, 2008

\author{
National Synchrotron Light Source II Project \\ Brookhaven National Laboratory \\ P.O. Box 5000 \\ Upton, NY 11973-5000 \\ www.bnl.gov
}

\begin{abstract}
Notice: This manuscript has been authored by employees of Brookhaven Science Associates, LLC under Contract No. DE-AC02-98CH10886 with the U.S. Department of Energy. The publisher by accepting the manuscript for publication acknowledges that the United States Government retains a non-exclusive, paid-up, irrevocable, world-wide license to publish or reproduce the published form of this manuscript, or allow others to do so, for United States Government purposes.
\end{abstract}




\section{DISCLAIMER}

This report was prepared as an account of work sponsored by an agency of the United States Government. Neither the United States Government nor any agency thereof, nor any of their employees, nor any of their contractors, subcontractors, or their employees, makes any warranty, express or implied, or assumes any legal liability or responsibility for the accuracy, completeness, or any third party's use or the results of such use of any information, apparatus, product, or process disclosed, or represents that its use would not infringe privately owned rights. Reference herein to any specific commercial product, process, or service by trade name, trademark, manufacturer, or otherwise, does not necessarily constitute or imply its endorsement, recommendation, or favoring by the United States Government or any agency thereof or its contractors or subcontractors. The views and opinions of authors expressed herein do not necessarily state or reflect those of the United States Government or any agency thereof. 


\title{
A theoretical study of the two-dimensional point focusing by two multilayer Laue lenses
}

\author{
Hanfei Yan ${ }^{\mathrm{a}, \mathrm{b}}$, Jorg Maser ${ }^{\mathrm{b}}$, Hyon Chol Kang ${ }^{\mathrm{b}, \mathrm{d}}$, Albert Macrander ${ }^{\mathrm{c}}$, and Brian Stephenson ${ }^{\mathrm{b}}$ \\ ${ }^{a}$ National Synchrotron Light Source II, Brookhaven National Laboratory, Upton, NY 11973 \\ ${ }^{\mathrm{b}}$ Center for Nanoscale Materials, Argonne National Laboratory, Argonne, IL 60439 \\ 'Advanced Photon Source, Argonne National Laboratory, Argonne, IL 60439 \\ ${ }^{\mathrm{d}}$ Advanced Photonics Research Institute, Gwangju Institute of Science and Technology, Gwangju \\ 500-712, Republic of Korea
}

\begin{abstract}
Hard x-ray point focusing by two crossed multilayer Laue lenses is studied using a full-wave modeling approach. This study shows that for a small numerical aperture, the two consecutive diffraction processes can be decoupled into two independent ones in respective directions. Using this theoretical tool, we investigated adverse effects of various misalignments on the 2D focus profile and discussed the tolerance to them. We also derived simple expressions that described the required alignment accuracy.
\end{abstract}

Keywords: hard x-ray, multilayer Laue lens, point focusing, misalignment

\section{INTRODUCTION}

Due to the great interest of high resolution x-ray microscopy in nanoscience, $x$-ray nanofocusing optics have drawn a lot of attentions recently. Sub-50 nm focus size has been achieved by K-B mirrors, ${ }^{1}$ refractive lenses, ${ }^{2}$ zone plates ${ }^{3,4}$ and multilayer Laue lenses (MLL's). ${ }^{5,6}$ Among these optics, MLL promises to deliver the true nanometer focus. Theoretical studies have shown that a focal size of 1-nm or even less can be achieved by MLL optics when wedged or curved structures are used. ${ }^{7}$ Other optics, however, possess a theoretical or practical limit that prevents x-rays from being focused to $1-\mathrm{nm}^{8,}{ }^{8}$ Since the first achievement of $30 \mathrm{~nm}$ line focus, ${ }^{5}$ there has been steady progress both in MLL fabrication and focus measurement. To date, a 16-nm line focus at energy of $19.5 \mathrm{keV}$ has been obtained, ${ }^{6}$ and the wedged MLL structure needed for 1-nm focus has been demonstrated. ${ }^{10}$ It is envisioned that MLL optics will produce the unprecedent 1-nm spot size at the planned National Synchrotron Light Source II, providing single atom detection ability. ${ }^{11}$

MLL is a one-dimensional focusing device, which only produces a line. To obtain a point focus that most of applications require, two MLL's have to be placed in series in a cross geometry, ${ }^{12}$ similar to a pair of K-B mirrors for point focusing. An obvious disadvantage of this set up is the efficiency: x-rays are absorbed twice in two lenses. Thanks to high focusing efficiency that an individual MLL can achieve, after two lenses a high efficiency up to $40 \%$ can still be achieved at optimum conditions. ${ }^{7}$ Another disadvantage of this crossed geometry is a more complicated mechanical design required to allow the line up of the two lenses: due to the short working distance of MLL ( mms), it is a mechanical challenge to accommodate many degrees of freedom in a small space. Thus, the knowledge of how a misalignment would affect the focusing performance of this optical system is very important. With this knowledge, we can make compromises accordingly and reduce the difficulty in the mechanical design while still achieve a same performance. In this paper we presented a full-wave modeling approach, based on the dynamical diffraction theory and Fourier optics, to study the focusing performance of two crossed MLL's. Using the theoretical tool, we investigated effects of misalignments on the point focus, and discussed the accuracy requirements. Simple expressions specifying the tolerance were derived. 


\section{THEORY}

We first consider a non-coplanar diffraction geometry for a single MLL as shown in Fig. 1 . The incident wave vector, $\vec{k}_{0}$, has components in three directions, $\vec{k}_{0}=k_{x} \vec{e}_{x}+k_{y} \vec{e}_{y}+k_{z} \vec{e}_{z}$, where

$$
k_{x}=k \cos \alpha \sin \theta, k_{y}=k \sin \alpha, k_{z}=k \cos \alpha \cos \theta \text {. }
$$

As an example we assume the MLL under consideration is composed by flat zones, so that the central differential equation governing the excitation of different diffraction orders [Eq. (12) in Ref. 6] becomes,

$$
\begin{gathered}
{\left[\left(\cos \alpha \sin \theta+\frac{h x}{f}\right) \frac{\partial}{\partial x}+\sin \alpha \frac{\partial}{\partial y}+\cos \alpha \cos \theta \frac{\partial}{\partial z}\right] E_{h}(x, y, z)=i \frac{\pi}{\lambda}\left[\beta_{h} E_{h}(x, y, z)+\sum_{l=-\infty}^{\infty} \chi_{h-l} E_{l}(x, y, z)\right]} \\
\beta_{h}=-2 \frac{h x}{f} \cos \alpha \sin \theta-\left(\frac{h x}{f}\right)^{2}, h=0, \pm 1, \pm 2, \ldots
\end{gathered}
$$

where $\lambda$ is the wavelength of the incident $\mathrm{x}$-ray, $f$ is the focal length and $\chi_{h}$ is the hth pseudo-Fourier coefficient of the MLL. The actual wavefield $E$ inside the MLL is a summation over all excitation orders,

$$
E=\sum_{h} E_{h} \exp \left[i \vec{k}_{0} \cdot \vec{r}+i \phi_{h}(x)\right], \phi_{h}=h \frac{\pi x^{2}}{\lambda f} .
$$

In general, $E_{h}$ is a function of coordinates $x, y$ and $z$. However, for an incident plane wave and an MLL with multilayers parallel to the $y z$ plane, it is obvious that $E_{h}\left(x, y_{1}, z\right)=E_{h}\left(x, y_{2}, z\right)$, assuming that the $y$ dimension is infinite. Therefore $\partial E_{h} / \partial y \equiv 0$ and Eq. (1) is simplified to,

$$
\left[\left(\cos \alpha \sin \theta+\frac{h x}{f}\right) \frac{\partial}{\partial x}+\cos \alpha \cos \theta \frac{\partial}{\partial z}\right] E_{h}(x, z)=i \frac{\pi}{\lambda}\left[\beta_{h} E_{h}(x, z)+\sum_{l=-\infty}^{\infty} \chi_{h-l} E_{l}(x, z)\right] .
$$

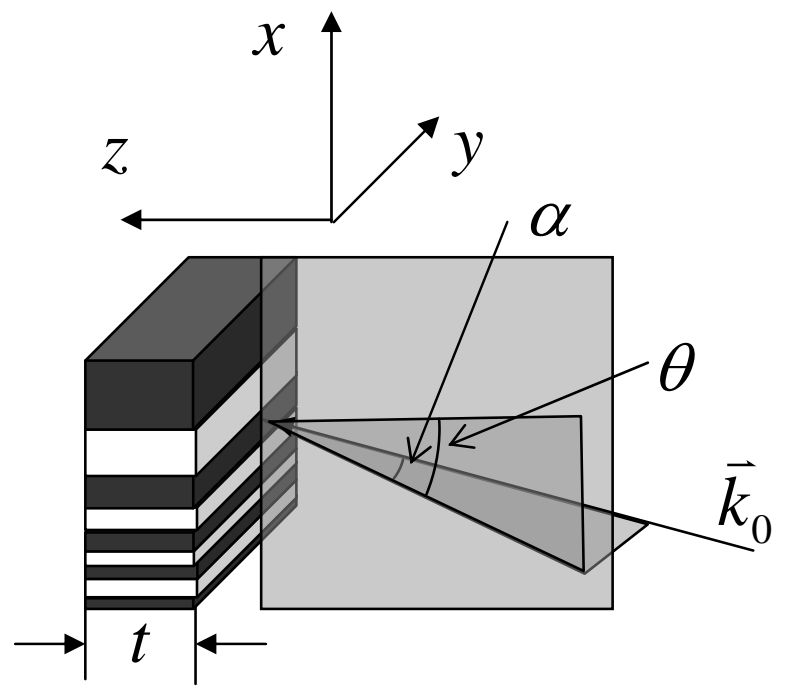

Fig. 1. An incident plane wave is diffracted by an MLL in the non-coplanar diffraction geometry.

Furthermore, for a small $\alpha$ we can take the approximation $\cos \alpha \approx 1$ (for example when $\alpha=1^{\circ}$, $\cos \alpha=0.99985$ ), which means that the non-coplanar solution is no different from the coplanar case if the titling angle, $\theta$, is the same, except that 
the fast varying phase term, $\exp \left[i \vec{k}_{0} \cdot \vec{r}+i \phi_{h}(x)\right]$, now contains a $y$-component. This approximation will lead to a much simplified calculation for a plane wave diffracted by two crossed MLL's, as shown below.

Let's consider the diffraction geometry shown in Fig. 2. An incident plane wave with wave vector $\vec{k}_{0}$ propagating along $z$ axis is diffracted vertically by MLL1, and then is diffracted horizontally by MLL2. We assume the two MLL's are separated by a distance $L_{1}$. The normal direction of the multilayer of MLL1, $\vec{n}_{1}$, lies in the $y z$ plane but have a tilting angle $\theta_{1}$ to the $y$-axis. The normal direction of the multilayer of MLL2, $\vec{n}_{2}$, has projected components in xyz coordinates system ( $\cos \gamma \cos \theta_{2}, \sin \gamma,-\cos \gamma \sin \theta_{2}$ ), where $\gamma$ corresponds to the in-plane misalignment angle. Ideally, we want this angle to be zero. The wavefield at a point $\vec{r}_{e_{1}}$ on the exit surface of MLL1 can be written as,

$$
E\left(\vec{r}_{e_{1}}\right)=\sum_{h_{1}} E_{1, h_{1}}\left(\vec{r}_{e_{1}}\right) \exp \left[i \vec{k}_{0} \cdot \vec{r}_{e_{1}}+i \phi_{1, h_{1}}\left(r_{1}\right)\right], \phi_{1, h_{1}}\left(r_{1}\right)=h_{1} \frac{\pi r_{1}^{2}}{\lambda f_{1}},
$$

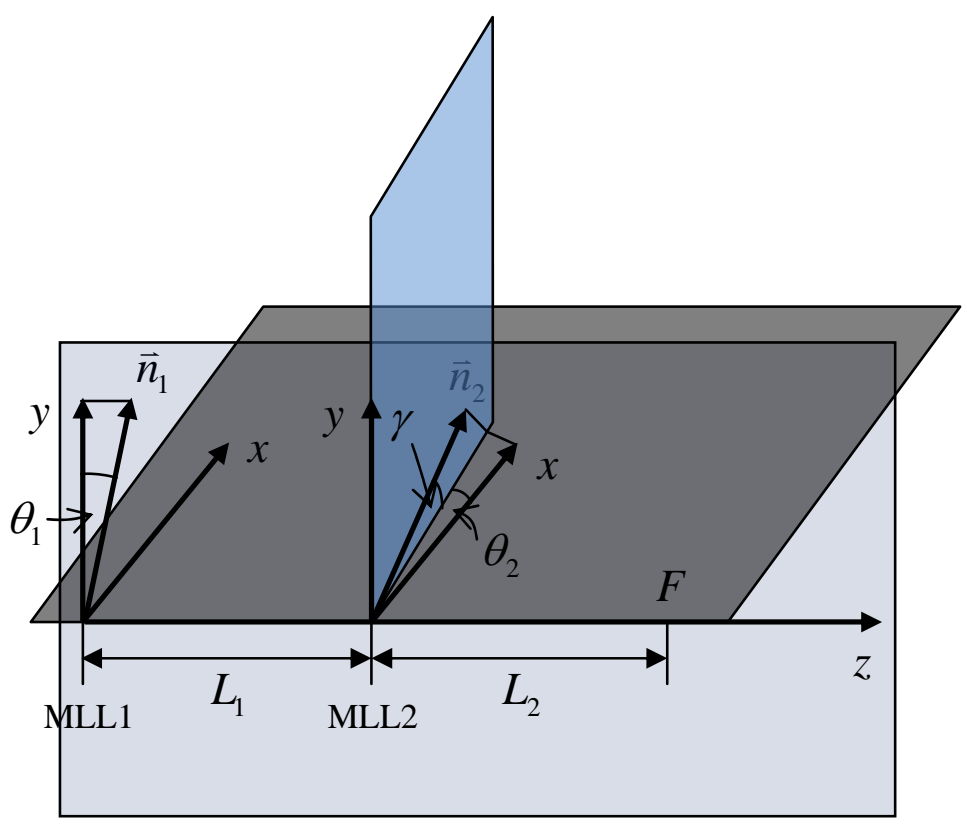

Fig. 2. The diffraction geometry and coordinates system of two crossed MLL's for point focusing at F. $\vec{n}_{1}$ and $\vec{n}_{2}$ are unit vectors along normal directions of the multilayers of MLL1 and MLL2, respectively.

where $E_{1, h_{1}}$ is the amplitude envelope function of the hth diffraction order excited by MLL1, $f_{1}$ is the focal length of MLL1, and $r_{1}$ is a variable along $\vec{n}_{1}$. This wavefield corresponds to a cylindrical wave, which propagates a distance of $L_{1}$ in free space to MLL2 and then is diffracted horizontally. To obtain the wavefield right after MLL2, we decompose this incident cylindrical wave to many plane waves whose wave vector $\vec{k}$ spans a fan with an angle $\alpha$ in $y z$ plane by performing Fourier transform,

$$
\varphi(\vec{k})=\frac{1}{2 \pi} \int_{-\infty}^{\infty} E(\vec{r}) \exp (-i \vec{k} \cdot \vec{r}) d y .
$$

For an incoming plane wave $\varphi(\vec{k}) \exp (\overrightarrow{i k} \cdot \vec{r})$, the resultant wavefield at a point $\vec{r}_{e_{2}}$ on the exit surface of MLL2 can be written as, 


$$
\varphi(\vec{k}) \exp \left(i \vec{k} \cdot \vec{r}_{e_{2}}\right) \sum_{h_{2}} E_{2, h_{2}} \exp \left[i \phi_{2, h_{2}}\left(r_{2}\right)\right], \phi_{2, h_{2}}\left(r_{2}\right)=h_{2} \frac{\pi r_{2}^{2}}{\lambda f_{2}},
$$

where $E_{2, h_{2}}$ is the amplitude envelope function of the hth diffraction order excited by MLL2, $f_{2}$ is the focal length, and $r_{2}$ is a variable along $\vec{n}_{2}$. If $\alpha$ is small (corresponds to a small numerical aperture), as we discussed previously the angle between different $\vec{k}$ and $\vec{n}_{2}$ is nearly unchanged and the solution is the same. As a result, we arrive at a simple expression of the wavefield on the exit surface of MLL2 after taking a summation over $\vec{k}$,

$$
\begin{aligned}
E\left(\vec{r}_{e_{2}}\right) & =\left\{\int_{-\infty}^{\infty} \varphi(\vec{k}) \exp \left(i \vec{k} \cdot \vec{r}_{e_{2}}\right) d k_{y}\right\}\left\{\sum_{h_{2}} E_{2, h_{2}} \exp \left[i \phi_{2, h_{2}}\left(r_{2}\right)\right]\right\} \\
& =V\left(\vec{r}_{e_{2}}\right) H\left(\vec{r}_{e_{2}}\right)
\end{aligned}
$$

where $V\left(\vec{r}_{e_{2}}\right)$ is the wavefield of the vertically diffracted wave as if there were no MLL2 and $H\left(\vec{r}_{e_{2}}\right)$ describes the horizontal diffraction by MLL2, independent of the vertical diffraction. Apparently, with the above approximation the whole diffraction process is decoupled into two independent ones in respective directions. After MLL2, the diffracted wave further propagates to the detector plane located at a distance $L_{2}$ downstream to the second lens. If $L_{2}$ is close to $f_{2}$, a point focus will form on the detector plane.

\section{SIMULATION}

Using Eq. (8), we can conduct a computer experiment and investigate the aberration induced by various misalignments. The two MLL's under considerations have the same parameters with the ones that have produced $30 \mathrm{~nm}$ and $16 \mathrm{~nm}$ line focus reported previously ${ }^{5,6}$. One has a focal length of $4.72 \mathrm{~mm}$ and outmost zone width of $9 \mathrm{~nm}$. The other one has a focal length of $2.6 \mathrm{~mm}$ and an outmost zone width of $5 \mathrm{~nm}$. Both of them correspond to about $40 \%$ of a full MLL; only half of the structure is fabricated and the central zones are not deposited. The MLL with a larger focal length is placed upstream to the other one and focuses x-ray vertically. The normal vector of its multilayers has a tilting angle $\theta_{1}=0.06^{\circ}$ to the $y$-axis. The second MLL which focuses x-rays horizontally, is placed at a distance of $L_{1}$ downstream to the first one, and has a tilting angle $\theta_{2}=0.15^{\circ}$ and a small in-plane misalignment angle $\gamma$, as shown in Fig. 2. The detector plane is assumed to be at a distance of $L_{2}$ downstream to the second MLL. For an incident plane wave at energy of $19.5 \mathrm{keV}$, in Fig. 3 we show the local diffraction efficiency of $-1^{\text {st }}$ order of each MLL at a section depth of $15 \mu \mathrm{m}$ and $10 \mu \mathrm{m}$, which will ideally produce a line focus $22 \mathrm{~nm}$ in vertical direction and $11 \mathrm{~nm}$ in horizontal direction. In the following we will study the change of the 2D focus profile with different misalignments.

Since the wavefield after two MLL's can be expressed as the product of the wavefields resulting from two independent diffraction processes, when $\gamma=0$ we expect that the tolerance of $L_{1}$ and $L_{2}$ are determined by the depth of focus in individual directions, which are $34 \mu \mathrm{m}$ and $10 \mu \mathrm{m}$ respectively. That is, $L_{1}+L_{2}$ cannot be $17 \mu \mathrm{m}$ larger or smaller than the nominal focal length of MLL1, and $L_{2}$ cannot be $5 \mu \mathrm{m}$ larger or smaller than the nominal focal length of MLL2 in order not to broaden the focus. In Fig. 4 we plot the intensity distribution of the 2D focal spot on the detector plane at different values of $L_{1}+L_{2}$ and $L_{2}$. Fig. 4a shows the focus profile when both MLL's are aligned perfectly. A peak width (full width of half maximum) of $22 \mathrm{~nm}$ in vertical direction and $11 \mathrm{~nm}$ in horizontal direction is observed. If we move the detector plane downstream by $10 \mu \mathrm{m}$, which increases both $L_{1}+L_{2}$ and $L_{2}$ by a same amount, the horizontal width of the focus peak is clearly broadened while its vertical shape remains unchanged. This is expected since this displacement is smaller than the tolerance of the focal distance in vertical direction but larger than that in horizontal direction. 


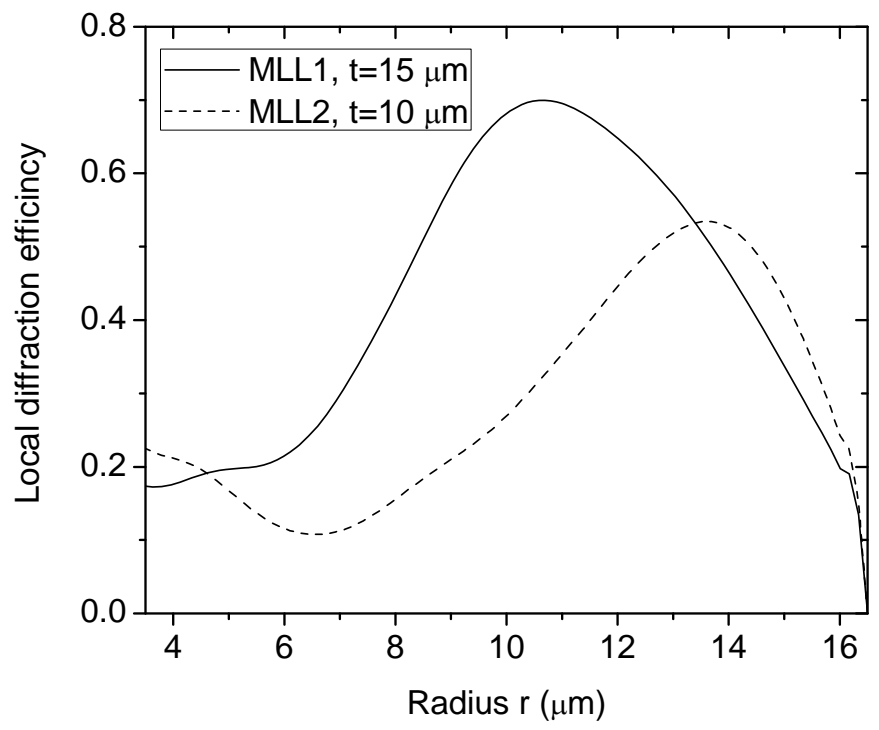

Fig. 3. Local diffraction efficiency of $-1^{\text {st }}$ diffraction order on the exit surface of MLL1 and MLL2.

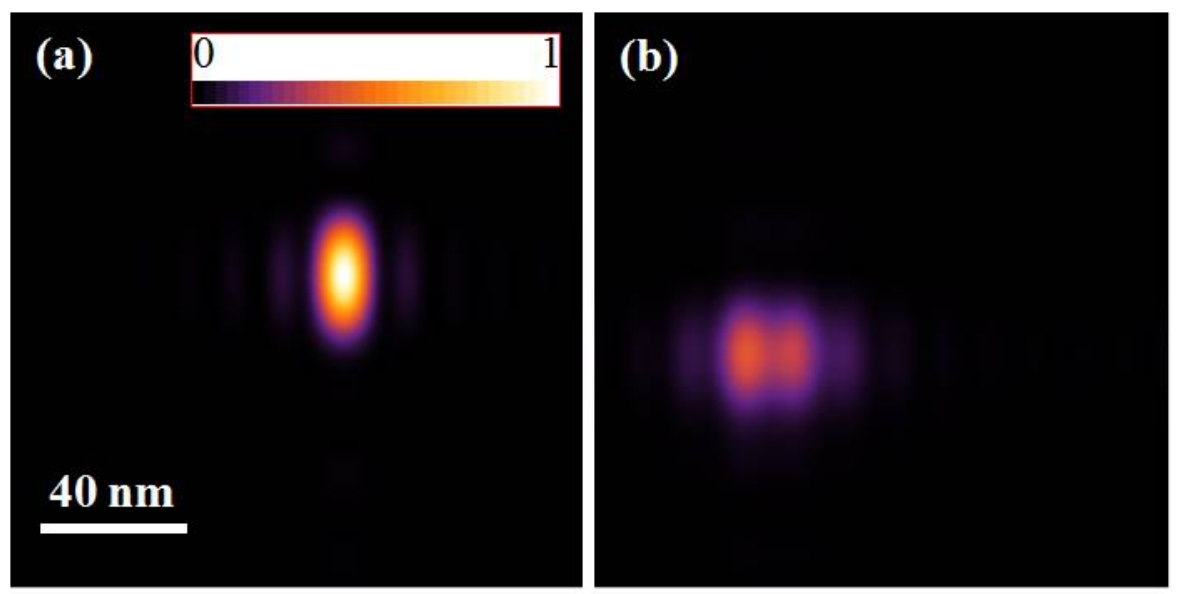

Fig. 4. 2D point focus profiles in case a) aligned perfectly and b) detector moves $10 \mu \mathrm{m}$ away from the best focal plane ( $\gamma=$ $0)$. Intensity is in linear scale.

The effect of the in-plane misalignment angle $\gamma$ on the focus is more subtle. In Fig. 5 we plot the 2D focus profiles when $\gamma=0^{\circ}, 0.05^{\circ}, 0.1^{\circ}$ and $0.15^{\circ}$, assuming that $L_{1}+L_{2}$ and $L_{2}$ are at the right values. We observe that a slight distortion occurs at $\gamma=0.05^{\circ}$, which only results in a very small broadening on peak width. The distortion becomes more pronounced as $\gamma$ increases as expected. At $\gamma=0.15^{\circ}$, the peak width is increased to $13 \mathrm{~nm}$ and $26 \mathrm{~nm}$, in $x$ and $y$ direction respectively. Therefore for this particular case, we want to control the in-plane misalignment angle within $0.05^{\circ}$. This result, at first glance, is a little bit surprising because from a "naive" point of view an MLL would only compress the dimension perpendicular to its multilayers and an in-plane misalignment angle would result in a projected uncompressed component, $D \sin \gamma$, of an incident line beam, where $D$ is the acceptance aperture of the MLL. If we put in numbers, for $D$ $=13 \mu \mathrm{m}$ and $\gamma=0.15^{\circ}$ the uncompressed component is $34 \mathrm{~nm}$ that is way larger than what we observe from the simulation. This disagreement can be understood by considering the simple case shown in Fig 6, where a line beam is incident on an MLL. If the MLL follows a zone plate law $x_{n}^{2}=n \lambda f$, the small angle $\gamma$ between $x$ and $x^{\prime}$ leads to a 

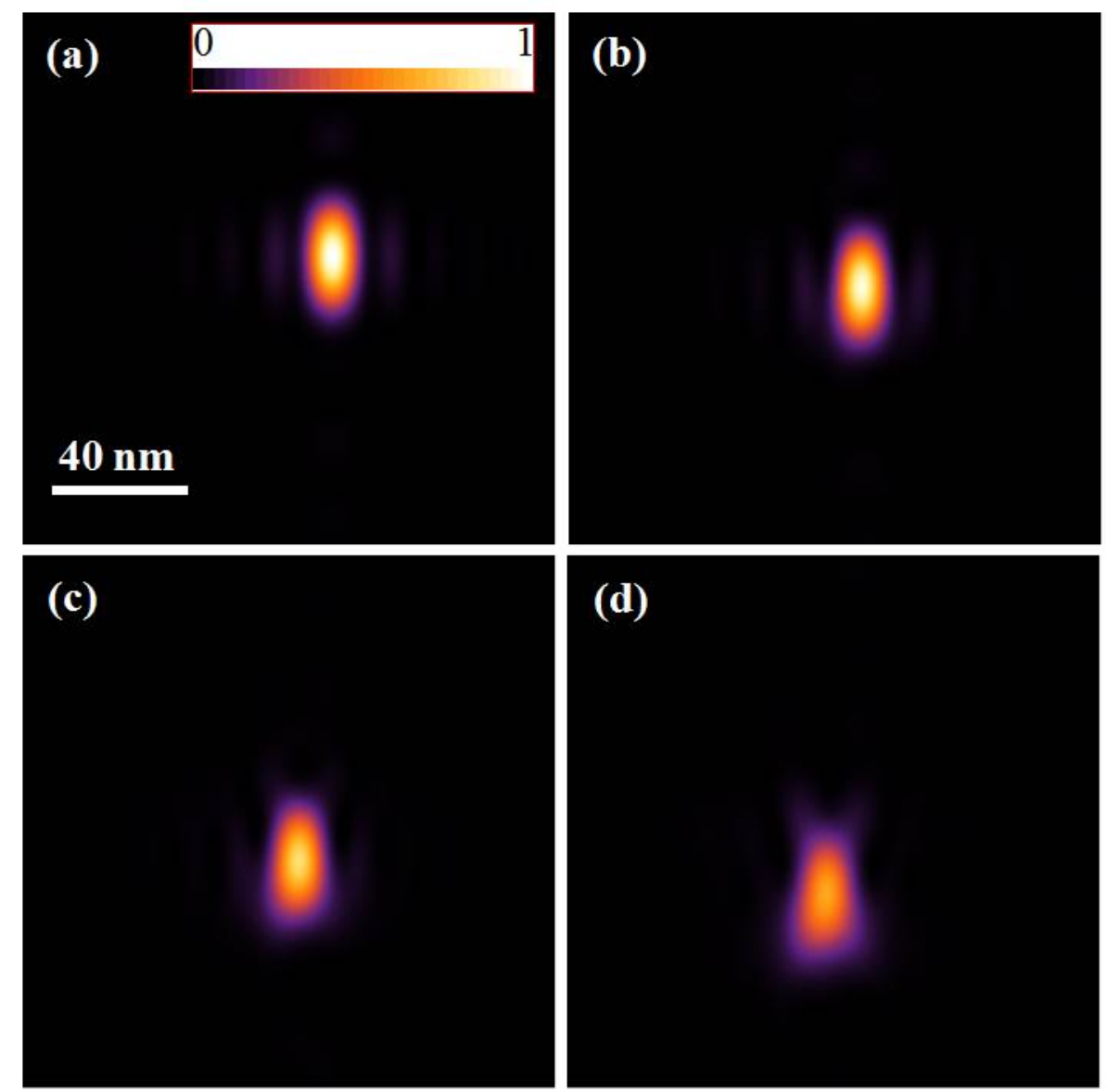

Fig. 5. 2D point focus profiles in case a) aligned perfectly, b) an in-plane misalignment angle $\gamma=0.05^{\circ}$, c) $0.1^{\circ}$ and d) $0.15^{\circ}$. We assume the detector plane is not moved. Intensity is in linear scale.

new zone plate law along the line, $x_{n}^{\prime 2}=n \lambda f^{\prime}$, where $x_{n}^{\prime}=x_{n} / \cos \gamma$. As a result, the line beam is compressed along $x^{\prime}$ instead of $x$, but with a focal length increased to $f^{\prime}=f / \cos ^{2} \gamma$. If $\gamma$ is small enough so that the change of the focal length is within the depth of focus, there will be no effect on the focus. That is, $\gamma<\sqrt{2 \lambda f / D^{2}}$. For an MLL with full structure, it can be written as $\gamma<\sqrt{1 / N}$, where $N$ is the total number of zones counting both sides. For the 2-D case, on the exit surface of MLL2 for a point located at $\vec{r}_{e_{2}}=\left(x, y, L_{1}-x \tan \theta_{2}\right)$ we have the relationship $r_{2}=x \cos \gamma / \cos \theta_{2}+y \sin \gamma$. In addition to the slight change of the focal length, there is a cross phase term $\exp \left(-i \frac{\pi \sin 2 \gamma}{\lambda f_{2} \cos \theta_{2}} x y\right)$ causing distortions. For it being negligible, the phase change associated with this term across the acceptance aperture has to be small enough. A good estimation of the maximum phase change allowed without causing a distortion is $\pi$, which yielding $\gamma<\lambda f_{2} / 2 D^{2}$. For a full structure, $\gamma<1 / 4 N$. For parameters discussed here, from this calculation we obtain $\gamma<0.03^{\circ}$, which agrees with the simulation. 


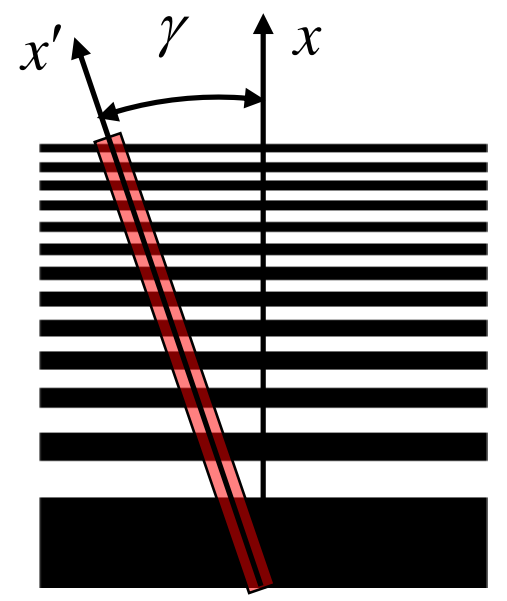

Fig. 6. A schematic drawing of a line beam incident on an MLL with an angle $\gamma$ to the normal direction of the multilayers.

\section{SUMMARY}

We presented a full-wave modeling approach to simulate the wave propagation of an incident plane wave diffracted by two crossed MLL's. We showed that for a small numerical aperture the whole diffraction process in two directions was independent. Using this simulation tool, we investigated the adverse effect of various misalignments on the point focus and discussed tolerances to them. Simple expressions specifying the alignment requirements were derived. This information can be used to guide the mechanical design of 2D point focusing MLL instrument.

\section{ACKNOWLEDGEMENT}

This work was supported by the U.S. Department of Energy, Office of Science, Office of Basic Energy Sciences, under Contract No.DE-AC-02-98CH10886 and DE-AC-02-06CH11357.

\section{REFERENCES}

[1] H. Mimura, H. Yumoto, S. Matsuyama, Y. Sano, K. Yamamura, Y. Mori, M. Yabashi, Y. Nishino, K. Tamasaku, T. Ishikawa and K. Yamauchi, Applied Physics Letters 90 (5), 051903 (2007).

[2] C. G. Schroer, O. Kurapova, J. Patommel, P. Boye, J. Feldkamp, B. Lengeler, M. Burghammer, C. Riekel, L. Vincze, A. van der Hart and M. Kuchler, Applied Physics Letters 87 (12), 124103 (2005).

[3] G.-C. Yin, Y.-F. Song, M.-T. Tang, F.-R. Chen, K. S. Liang, F. W. Duewer, M. Feser, W. Yun and H.-P. D. Shieh, Applied Physics Letters 89 (22), 221122 (2006).

[4] Y. S. Chu, J. M. Yi, F. De Carlo, Q. Shen, W. K. Lee, H. J. Wu, C. L. Wang, J. Y. Wang, C. J. Liu, C. H. Wang, S. R. Wu, C. C. Chien, Y. Hwu, A. Tkachuk, W. Yun, M. Feser, K. S. Liang, C. S. Yang, J. H. Je and G. Margaritondo, Applied Physics Letters 92 (10), 103119 (2008).

[5] H. C. Kang, J. Maser, G. B. Stephenson, C. Liu, R. Conley, A. T. Macrander and S. Vogt, Physical Review Letters 96 (12), 127401 (2006).

[6] H. C. Kang, H. F. Yan, R. P. Winarski, M. V. Holt, J. Maser, C. A. Liu, R. Conley, S. Vogt, A. T. Macrander and G. B. Stephenson, Applied Physics Letters 92 (22), 221114 (2008).

[7] H. F. Yan, J. Maser, A. Macrander, Q. Shen, S. Vogt, G. B. Stephenson and H. C. Kang, Physical Review B 76 (11), 115438 (2007).

C. Bergemann, H. Keymeulen and J. F. van der Veen, Physical Review Letters 91 (20), 204801-204804 (2003).

[9] C. G. Schroer and B. Lengeler, Physical Review Letters 94 (5), 054802 (2005). 
[10] R. Conley, C. Liu, J. Qian, C. M. Kewish, A. T. Macrander, H. Yan, H. C. Kang, J. Maser and G. B. Stephenson, Review of Scientific Instruments 79 (5), 053104 (2008).

[11] S. Dierker et al. NSLS II Conceptual Design Report, Brookhaven National Laboratory, December 2006; http://www.bnl.gov/nsls2/project/CDR.

[12] J. Maser, G. B. Stephenson, S. Vogt, Y. Wenbing, A. Macrander, H. C. Kang, L. Chian and R. Conley, Proceedings of the SPIE - The International Society for Optical Engineering 5539 (1), 185-194 (2004). 